\title{
BMJ Open Interventions to improve the quality of cataract services: protocol for a global scoping review
}

\author{
Miho Yoshizaki (D) , ${ }^{1}$ Jacqueline Ramke (D) , ${ }^{1,2}$ João M Furtado, ${ }^{3}$ Helen Burn, ${ }^{4}$ \\ Stephen Gichuhi, ${ }^{5}$ Iris Gordon, ${ }^{1}$ Ada Aghaji, ${ }^{6}$ Ana P Marques (D),${ }^{1}$ \\ William H Dean, ${ }^{1,7}$ Nathan Congdon, ${ }^{8,9}$ John Buchan, ${ }^{1}$ Matthew J Burton ${ }^{1,10}$
}

To cite: Yoshizaki M, Ramke J, Furtado JM, et al. Interventions to improve the quality of cataract services: protocol for a global scoping review. BMJ Open 2020;10:e036413. doi:10.1136/ bmjopen-2019-036413

- Prepublication history and additional material for this paper are available online. To view these files, please visit the journal online (http://dx.doi. org/10.1136/bmjopen-2019036413).

Received 13 December 2019 Revised 15 April 2020 Accepted 23 April 2020

Check for updates

(C) Author(s) (or their employer(s)) 2020. Re-use permitted under CC BY. Published by BMJ.

For numbered affiliations see end of article.

Correspondence to

Miho Yoshizaki;

miho.yoshizaki@nhs.net

\section{ABSTRACT}

Introduction Cataract is the leading cause of blindness globally and a major cause of vision impairment. Cataract surgery is an efficacious intervention that usually restores vision. Although it is one of the most commonly conducted surgical interventions worldwide, good quality services (from being detected with operable cataract to undergoing surgery and receiving postoperative care) are not universally accessible. Poor quality understandably reduces the willingness of people with operable cataract to undergo surgery. Therefore, it is critical to improve the quality of care to subsequently reduce vision loss from cataract. This scoping review aims to summarise the nature and extent of the published literature on interventions to improve the quality of services for primary age-related cataract globally.

Methods and analysis We will search MEDLINE, Embase and Global Health for peer-reviewed manuscripts published since 1990, with no language, geographic or study design restrictions. To define quality, we have used the elements adopted by the $\mathrm{WHO}$ - effectiveness, safety, people-centredness, timeliness, equity, integration and efficiency - to which we have added the element of planetary health. We will exclude studies focused on the technical aspects of the surgical procedure and studies that only involve children ( $<18$ years). Two reviewers will screen all titles/abstracts independently, followed by a full-text review of potentially relevant articles. For included articles, data regarding publication characteristics, study details and quality-related outcomes will be extracted by two reviewers independently. Results will be synthesised narratively and presented visually using a spider chart. Ethics and dissemination Ethical approval was not sought, as our review will only include published and publicly accessible information. We will publish our findings in an open-access peer-reviewed journal and develop an accessible summary of the results for website posting. A summary of the results will be included in the ongoing Lancet Global Health Commission on Global Eye Health.

Registration details Open Science Framework (https:// osf.io/8gktz).

\section{INTRODUCTION}

Cataract is the leading cause of blindness globally and a major cause of moderate and severe

\section{Strengths and limitations of this study}

- A strength of this review is the use of a broader concept of quality beyond the common measure of postoperative visual acuity-we included the seven elements of quality outlined in WHO's framework for healthcare quality, as well as the element of planetary health.

- Another strength is that we have broadened the scope of cataract services beyond the surgical intervention itself to identify interventions to improve quality along the care pathways, from detection and referral to uptake of services through to postoperative care.

- This study will not include studies that assess specific surgical techniques and/or specific products and medications as this extensive literature is commonly synthesised in Cochrane and other reviews.

- This review will summarise the nature and extent of the literature on interventions to improve the quality of cataract services but will not assess the quality or risk of bias of the studies themselves.

vision impairment-an estimated 65 million people had vision loss from cataract in $2015 .{ }^{1}$ Vision loss from cataract is unequally distributed throughout the world. For example, in 2015, among adults aged 50 years and above, the age-standardised prevalence of cataract blindness ranged from $0.08 \%(80 \%$, uncertainty interval (UI) $0.03 \%-0.19 \%$ ) in highincome countries of the Asia Pacific region to $2.35 \%(80 \%$, UI $0.72 \%-5.04 \%)$ in West sub-Saharan Africa-almost a 30-fold difference. ${ }^{1}$ Inequality (ie, measurable differences between population subgroups) is also evident within countries, with a higher prevalence of cataract blindness among socially disadvantaged groups such as women, rural dwellers and those who are not literate. ${ }^{2}$

Cataract surgery is an efficacious intervention that can restore vision ${ }^{3-5}$ and alleviate poverty. ${ }^{6} \mathrm{It}$ is one of the most common surgical interventions in many high-income 
countries and some middle-income countries. ${ }^{7}$ However, good quality services are not universally accessible, particularly in low/middle-income countries (LMICs). ${ }^{89}$ Poor quality understandably reduces the willingness of people with operable cataract to undergo surgery. ${ }^{10}$ Therefore, it is critical to improve the quality of care to subsequently reduce vision loss from cataract.

Quality of cataract services is most commonly measured using postoperative visual acuity. Measuring and monitoring outcomes is crucial in order to improve them ${ }^{11}$ and tools are available to enable monitoring of postoperative visual acuity. ${ }^{12}$

Beyond using postoperative visual acuity to assess effectiveness, the quality of cataract services includes many clinical and non-clinical dimensions. ${ }^{13}$ For example:

- Timeliness: cataract commonly occurs bilaterally. In many settings, the current recommendation is to operate on one eye at a time and allow enough time for the operated eye to heal before operating on the second eye. However, delay in surgery for the second eye has been linked to increased risk of falls and road traffic accidents. ${ }^{14}$

- People-centredness: it may be common for patients to have to visit hospitals several times before the surgery for different preoperative assessments, even though some of these could be done in one visit. Reducing the number of hospital visits to get surgery would improve quality from the patient perspective.

- Equity: there is no physiological reason why outcomes should be poorer in women compared with men, but women tend to have lower access and poorer postoperative vision outcomes compared with men. ${ }^{215}$ A further example of inequity is seen in the difference in effective cataract surgical coverage among indigenous (51.6\%, 95\% CI: 42.4-60.7) and non-indigenous Australians $(88.5 \%, 95 \% \mathrm{CI}$ : 85.2-91.2). ${ }^{16}$

- Efficiency (productivity): there is a link between the quantity of surgery a surgeon performs and the quality of that surgery. ${ }^{17}$ It has also been demonstrated that apparently cheaper service delivery options, such as outreach camps, can be less cost-effective compared with surgery delivered in static clinics due to worse outcomes. $^{18}$

The aim of this review is to summarise the nature and extent of the published literature on interventions to improve the quality of cataract services globally. We chose to undertake a scoping review rather than an alternative evidence synthesis approach because we wished to identify and map the available evidence, which we anticipate will be heterogeneous. ${ }^{19}{ }^{20}$ We will take a broad perspective on quality outcomes and relevant interventions of interest, but will exclude studies focused exclusively on the technical aspects of surgical techniques. For example, we will not include studies reporting the effectiveness of phacoemulsification or manual small incision surgery, as these are summarised in other reviews. ${ }^{3-5} 21$

\section{Definitions and framework development}

Cataract services include the range of activities on the pathway from detecting people with operable cataract to these people undergoing surgery and receiving postoperative care. As such, cataract services are both community and facility-based ${ }^{22}$ and-regardless of the settingshould involve a broad range of healthcare providers from the community level (eg, village health workers as case finders) through primary (eg, optometrist) and secondary services (ie, surgical team). In addition, consideration of all of the health system building blocks is relevant to strengthen cataract services.

Quality of care is one of the objectives embodied by the concept of Universal Health Coverage, together with equity in access and financial protection. ${ }^{23}$ Our review will be guided by the definition of the quality of care recently outlined by the WHO:

Quality of care is 'the degree to which health services for individuals and populations increase the likelihood of desired health outcomes and are consistent with current professional knowledge'. ${ }^{24}$

WHO has adopted the framework of quality outlined by the Institute of Medicine. ${ }^{25}$ This framework measures the quality of healthcare across seven elements, namely, effectiveness, safety, people-centredness, timeliness, equity, integration and efficiency.

We have made one addition to the quality elements in WHO's framework-we believe that planetary health is an essential element of quality cataract surgery, so will also scope the literature on this. Planetary health is focused on sustainability, including the ability of the society to make choices while balancing the needs of future generations. ${ }^{26}$ This modified framework is shown in figure 1 .

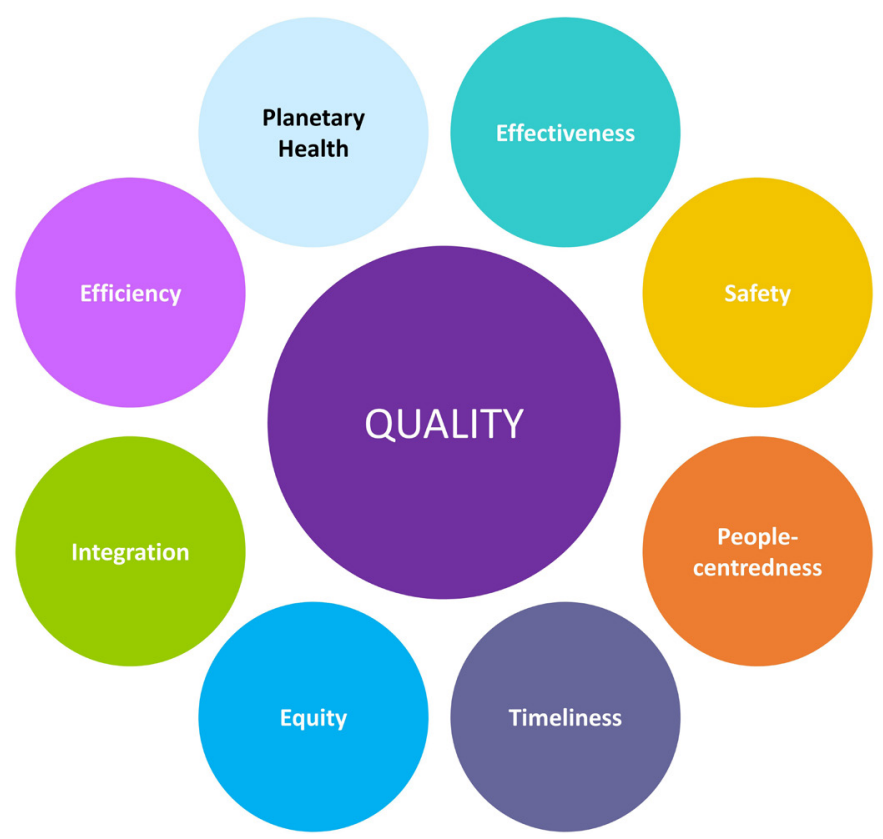

Figure 1 Elements of healthcare quality considered in this review (modified figure 3.2 from World Health Organization ${ }^{24}$ by adding planetary health). 
To help guide the scope of our review, we mapped examples of outcome measures and interventions for cataract services against each of the eight elements of healthcare quality (table 1 ). These outcomes and interventions were drawn from the literature, ${ }^{27} 28$ as well as the knowledge and experience of the authorship group. For people-centredness, we drew on the outline of Integrated Person-Centred Health Services provided by WHO and adopted in the recent World Report on Vision, whereby services aim to provide coordinated care that addresses the full spectrum of eye conditions according to an individual's needs and recognises people as participants and beneficiaries of this care. ${ }^{29} 30$

When mapping interventions, we categorised them using the WHO health systems 'building blocks', that is, we mapped them to the most relevant of service delivery; health workforce/human resources (HR), health information system (HIS); access to essential consumables/ non-consumables; financing; and leadership/governance. Recognising that this framework does not include community engagement and empowerment, we added community as an additional category against which interventions could be mapped. ${ }^{31}$

\section{METHODS AND ANALYSIS}

\section{Objectives/scoping review questions}

We aim to answer the following three questions:

1. What interventions to improve the quality of cataract services have been described in the published literature?

2. Which element(s) of quality did the interventions address?

3. Where was the evidence generated (high-income vs middle-income vs low-income settings)?

\section{Protocol and registration}

This protocol for this scoping review is reported according to the relevant sections of the Preferred Reporting Items for Systematic Reviews and Meta-Analyses Extension for Scoping Reviews (PRISMA-ScR) guideline (online supplementary annex 1). ${ }^{32}$

\section{Eligibility criteria}

This scoping review will include primary research studies of any design and systematic reviews from any country that report a quality-relevant outcome for primary age-related cataract following an intervention related to the quality of cataract services. We will only include studies where intervention is compared against any alternatives (eg, intervention vs no intervention/current practice vs new intervention/before vs after implementation). Examples of relevant interventions are provided in table 1, mapped against the eight quality elements of interest. Systematic reviews will be included only if meta-analysis is conducted for a quality-relevant outcome. If we identify systematic reviews that report narrative synthesis of quality-relevant outcomes without meta-analysis, then we will review the list of included studies and include in our scoping review any that meets our eligibility criteria.

We will exclude studies assessing specific surgical techniques (eg, phacoemulsification vs manual small incision surgery, site of anaesthesia and size of incision) and/ or specific products and medications used during and around the time of surgery (eg, monofocal vs multifocal intraocular lens; drug A vs drug B) as these are typically addressed in other systematic reviews. ${ }^{3421}$ Studies focused exclusively on cataract services for children (aged under 18 years) will be excluded, as these services differ substantially from those for age-related cataract. We will also exclude studies reporting interventions to prevent cataract formation or progression. We will exclude studies published prior to 1990, as during the last 30 years, there have been a large number of major developments in cataract services that would be expected to have changed the 'landscape' substantially. Service delivery models prior to this time are quite different from those currently used. There will be no language limitations. Only studies where the full text is available will be included.

\section{Search}

We will search MEDLINE, Embase and Global Health databases using search strategies developed by a Cochrane Eyes and Vision Information Specialist (IG). The search strategy for MEDLINE is included in the online supplementary annex 2. We will examine reference lists of all included articles to identify further potentially relevant reports of studies. Field experts will be provided a list of the included studies and requested to identify further potentially relevant studies for consideration in the review.

\section{Selection of sources of evidence}

Covidence systematic review software will be used for screening (Veritas Health Innovation, Melbourne, Australia. Available at: www.covidence.org). Each title and abstract will be screened independently by two reviewers (MY, JR, HB, AA, JB, JF, SG and WHD) to exclude publications that clearly do not meet the inclusion criteria. Subsequently, the full-text article will be retrieved for review if the citation seems potentially relevant and two reviewers will independently assess each article against the inclusion and exclusion criteria. Any discrepancies between the reviewers will be resolved by discussion and a third reviewer will be consulted if necessary. A PRISMA flow diagram will be completed to summarise the study selection process.

\section{Data charting process}

A custom form will be developed in Excel for data charting. The form will be piloted on three studies and required amendments agreed by consensus. We anticipate a broad scope of included studies, so data charting will be an iterative process throughout the review and the data charting form will be amended as required. Each included study will be charted independently by two 


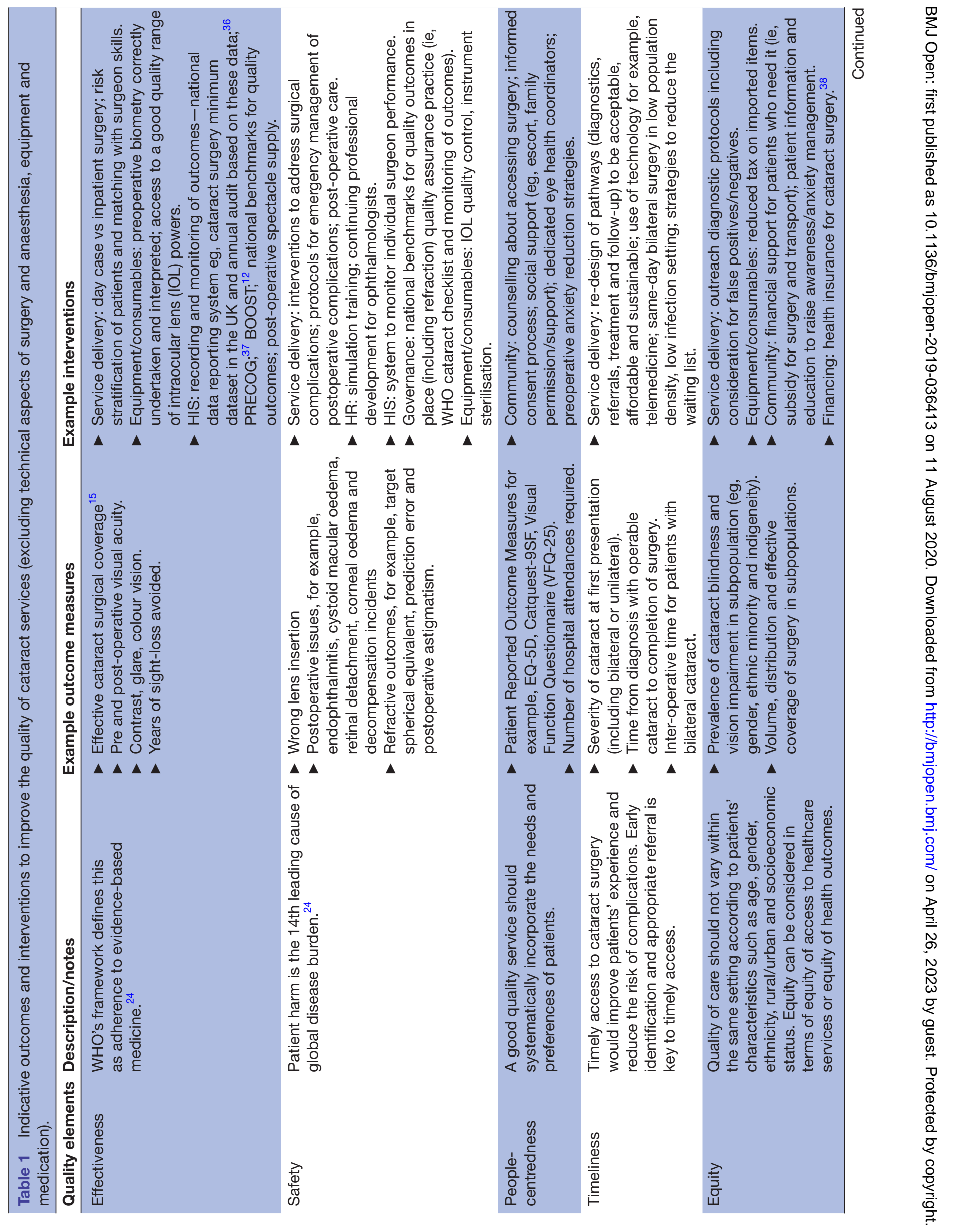




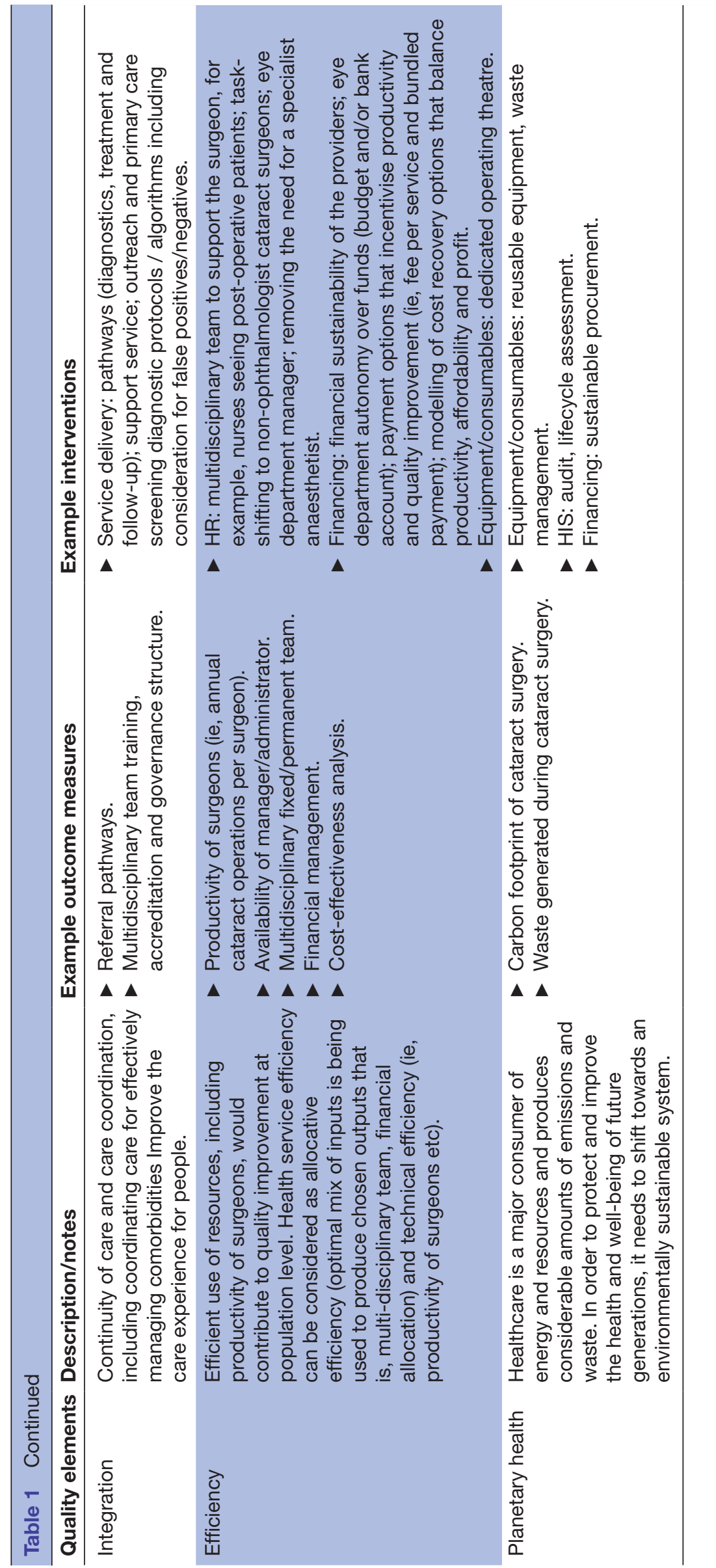


reviewers. Any discrepancies will be resolved by discussion and a third reviewer will be consulted if necessary. We plan to contact study authors in the case of unclear information and will make up to three attempts by email.

\section{Data items}

The following data items will be collected during the data charting process:

1. Publication characteristics: title, year of publication, study design, country of origin and study setting.

2. Characteristics of intervention/study:

a. Context (eg, geographic area, target population and distribution, type of interventions (categorised by health system building block), target health practitioner and duration/frequency).

b. Quality element(s) addressed by the intervention (as outlined in table 1).

3. Outcome(s) of the intervention/study and whether it was reported to be effective (ie, had an effect vs had no effect) (examples of outcomes are outlined in table 1).

\section{Synthesis of results}

We recognise that the indication for surgery can vary across different settings due to the prevalence of vision loss from cataract, the capacity of services and the quality and safety standards in each setting. Accordingly, we will synthesise results by World Bank country income level (high/upper-middle/lower-middle/low) ${ }^{33}$ and (if possible) by Global Burden of Disease super-region (high income/Latin America and Caribbean/sub-Saharan Africa/North Africa and Middle East/Southeast Asia, East Asia and Oceania/South Asia/Central Europe, Eastern Europe and Central Asia). ${ }^{34}$

We will summarise findings narratively and using descriptive statistical methods as appropriate. We will map each intervention to the relevant quality element. We will visualise the findings using spider charts to show the extent of the evidence across each quality element and will plot evidence in high-income countries separately to LMICs. For each intervention, we will quantify the number of studies that were reported by the authors to be effective (vs having no effect).

\section{Patient and public involvement statement}

This protocol was developed with input from the Commissioners of the Lancet Global Health Commission on Global Eye Health, ${ }^{35}$ which includes people with lived experience of vision impairment (and cataract surgery), policymakers, academics, clinicians, government eye health programme leaders and advocacy specialists.

\section{ETHICS AND DISSEMINATION}

Ethical approval was not sought, as our review will only include published and publicly accessible information.

We will publish our findings in an open-access, peerreviewed journal and develop an accessible summary of the results for website posting and stakeholder meetings.
A summary of the results will also be included in the ongoing Lancet Global Health Commission on Global Eye Health. ${ }^{35}$

Author affiliations

${ }^{1}$ International Centre for Eye Health, London School of Hygiene and Tropical

Medicine, London, UK

${ }^{2}$ School of Optometry and Vision Science, The University of Auckland, Auckland, New Zealand

${ }^{3}$ Division of Ophthalmology, Universidade de São Paulo, Faculdade de Medicina de Ribeirão Preto, Ribeirao Preto, São Paulo, Brazil

${ }^{4}$ Department of Ophthalmology, Stoke Mandeville Hospital, Aylesbury, UK

${ }^{5}$ Department of Ophthalmology, University of Nairobi, Nairobi, Kenya

${ }^{6}$ Department of Ophthalmology, University of Nigeria, Nsukka, Enugu, Nigeria

${ }^{7}$ Department of Ophthalmology, University of Cape Town, Rondebosch, Western Cape, South Africa

${ }^{8}$ Centre for Public Health, Queen's University Belfast, Belfast, UK

${ }^{9}$ Zhongshan Ophthalmic Center, Sun Yat-Sen University, Guangzhou, Guangdong, China

${ }^{10}$ Moorfields Eye Hospital, London, UK

Contributors JR and MJB conceived the idea for the review. MY and JR drafted and revised the protocol with suggestions from MJB, NC, JF, SG, HB, AA, APM, WHD and JB. IG constructed the search.

Funding MJB is supported by the Wellcome Trust (207472/Z/17/Z). JR is a Commonwealth Rutherford Fellow, funded by the UK government through the Commonwealth Scholarship Commission in the UK. The Lancet Global Health Commission on Global Eye Health is supported by The Queen Elizabeth Diamond Jubilee Trust, Moorfields Eye Charity [grant number GR001061], NIHR Moorfields Biomedical Research Centre, Wellcome Trust, Sightsavers, The Fred Hollows Foundation, The SEVA Foundation, British Council for the Prevention of Blindness and Christian Blind Mission.

Competing interests None declared.

Patient and public involvement Patients and/or the public were involved in the design, or conduct, or reporting, or dissemination plans of this research. Refer to the Methods section for further details.

Patient consent for publication Not required.

Provenance and peer review Not commissioned; externally peer reviewed.

Open access This is an open access article distributed in accordance with the Creative Commons Attribution 4.0 Unported (CC BY 4.0) license, which permits others to copy, redistribute, remix, transform and build upon this work for any purpose, provided the original work is properly cited, a link to the licence is given, and indication of whether changes were made. See: https://creativecommons.org/ licenses/by/4.0/.

\section{ORCID iDs}

Miho Yoshizaki http://orcid.org/0000-0003-1893-5675

Jacqueline Ramke http://orcid.org/0000-0002-5764-1306

Ana P Marques http://orcid.org/0000-0001-8242-7021

\section{REFERENCES}

1 Flaxman SR, Bourne RRA, Resnikoff S, et al. Global causes of blindness and distance vision impairment 1990-2020: a systematic review and meta-analysis. Lancet Glob Health 2017;5:e1221-34.

2 Ramke J, Zwi AB, Lee AC, et al. Inequality in cataract blindness and services: moving beyond unidimensional analyses of social position. Br J Ophthalmol 2017;101:395-400.

3 de Silva SR, Riaz Y, Evans JR. Phacoemulsification with posterior chamber intraocular lens versus extracapsular cataract extraction (ECCE) with posterior chamber intraocular lens for age-related cataract. Cochrane Database Syst Rev 2014:CD008812.

4 Ang M, Evans JR, Mehta JS, et al. Manual small incision cataract surgery (MSICS) with posterior chamber intraocular lens versus extracapsular cataract extraction (ECCE) with posterior chamber intraocular lens for age-related cataract. Cochrane Database Syst Rev 2014;12:CD008811.

5 Riaz Y, de Silva SR, Evans JR. Manual small incision cataract surgery (MSICS) with posterior chamber intraocular lens 
versus phacoemulsification with posterior chamber intraocular lens for age-related cataract. Cochrane Database Syst Rev 2013:CD008813.

6 Kuper H, Polack S, Mathenge W, et al. Does cataract surgery alleviate poverty? Evidence from a multi-centre intervention study conducted in Kenya, the Philippines and Bangladesh. PLoS One 2010;5:e15431.

7 Eurostat. Surgical operations and procedures statistics. Available: https://ec.europa.eu/eurostat/statistics-explained/index.php?title= Surgical_operations_and_procedures_statistics [Accessed 15 Nov 2019].

8 Zhao J, Xu X, Ellwein LB, et al. Cataract surgical coverage and visual acuity outcomes in rural China in 2014 and comparisons with the 2006 China Nine-Province survey. Am J Ophthalmol 2018;193:62-70.

9 Silva JC, Mújica OJ, Vega $\mathrm{E}$, et al. A comparative assessment of avoidable blindness and visual impairment in seven Latin American countries: prevalence, coverage, and inequality. Rev Panam Salud Publica 2015;37:13-20.

10 Yin Q, Hu A, Liang Y, et al. A two-site, population-based study of barriers to cataract surgery in rural China. Invest Ophthalmol Vis Sci 2009;50:1069-75

11 Yorston D, Gichuhi S, Wood M, et al. Does prospective monitoring improve cataract surgery outcomes in Africa? Br J Ophthalmol 2002;86:543-7.

12 Congdon N, Suburaman G-B, Ravilla T, et al. Transforming research results into useful tools for global health: boost. Lancet Glob Health 2016;4:e96.

13 Donabedian A. The quality of care. How can it be assessed? JAMA 1988;260:1743-8.

14 Meuleners LB, Fraser ML, Ng J, et al. The impact of first- and second-eye cataract surgery on injurious falls that require hospitalisation: a whole-population study. Age Ageing 2014;43:341-6.

15 Ramke J, Gilbert CE, Lee AC, et al. Effective cataract surgical coverage: an indicator for measuring quality-of-care in the context of universal health coverage. PLoS One 2017;12:e0172342.

16 Keel S, Xie J, Foreman J, et al. Population-Based assessment of visual acuity outcomes following cataract surgery in Australia: the National eye health survey. Br J Ophthalmol 2018;102:1419-24.

17 Bell CM, Hatch WV, Cernat G, et al. Surgeon volumes and selected patient outcomes in cataract surgery: a population-based analysis. Ophthalmology 2007;114:405-10.

18 Singh AJ, Garner P, Floyd K. Cost-Effectiveness of public-funded options for cataract surgery in Mysore, India. Lancet 2000;355:180-4.

19 Joanna Briggs Institute. JBI reviewer's manual, 2019. Available: https://wiki.joannabriggs.org/display/MANUAL [Accessed 9 Mar 2020].

20 Munn Z, Peters MDJ, Stern C, et al. Systematic review or scoping review? guidance for authors when choosing between a systematic or scoping review approach. BMC Med Res Methodol 2018;18:143.
21 Riaz Y, Mehta J, Wormald R, et al. Surgical interventions for agerelated cataract. Cochrane Database Syst Rev 2006;4.

22 World Health Organization. Strategies for the prevention of blindness in national programmes: a primary health care approach. 2nd ed. Geneva: World Health Organization, 1997.

23 World Health Organization. What is universal coverage? 2016. Available: http://www.who.int/health_financing/universal_coverage_ definition/en/ [Accessed 1 Aug 2017]

24 World Health Organization. Delivering quality health services: a global imperative for universal health coverage. Geneva: WHO, 2018.

25 Institute of Medicine Committee on Health Care in America. Crossing the quality chasm: a new health system for the 21st century. Washington DC: National Academies Press, 2001.

26 The Lancet Planetary Health. The bigger picture of planetary health. Lancet Planet Health 2019;3:e1.

27 Buchan JC, Dean WH, Foster A, et al. What are the priorities for improving cataract surgical outcomes in Africa? results of a Delphi exercise. Int Ophthalmol 2018;38:1409-14.

28 Lindfield R, Vishwanath K, Ngounou F, et al. The challenges in improving outcome of cataract surgery in low and middle income countries. Indian J Ophthalmol 2012;60:464.

29 World Health Organization. Framework on integrated, people-centred health services. Geneva: WHO, 2016.

30 World Health Organization. World report on vision. Geneva: WHO, 2019.

31 Sacks E, Morrow M, Story WT, et al. Beyond the building blocks: integrating community roles into health systems frameworks to achieve health for all. BMJ Glob Health 2018;3:e001384.

32 Tricco AC, Lillie E, Zarin W, et al. PRISMA extension for scoping reviews (PRISMA-ScR): checklist and explanation. Ann Intern Med 2018;169:467-73.

33 World Bank. Country and lending groups. Available: https:// datahelpdesk.worldbank.org/knowledgebase/articles/906519-worldbank-country-and-lending-groups [Accessed 2 Nov 2019].

34 Institute for Health Metrics and Evaluation. Frequently asked questions - what countries are in each regions?Available: https:// datahelpdesk.worldbank.org/knowledgebase/articles/906519-worldbank-country-and-lending-groups [Accessed 15 Nov 2019].

35 Burton MJ, Faal HB, Ramke J, et al. Announcing the Lancet global health Commission on global eye health. Lancet Glob Health 2019;7:e1612-3

36 Day AC, Donachie PHJ, Sparrow JM, et al. The Royal College of ophthalmologists' national ophthalmology database study of cataract surgery: report 1, visual outcomes and complications. Eye 2015;29:552-60.

37 Congdon N, Yan X, Lansingh V, et al. Assessment of cataract surgical outcomes in settings where follow-up is poor: PRECOG, a multicentre observational study. Lancet Glob Health 2013;1:e37-45.

38 Ackuaku-Dogbe EM, Yawson AE, Biritwum RB. Cataract surgical uptake among older adults in Ghana. Ghana Med J 2015;49:84-9. 